6095

March, 1993

$\mathrm{T} / \mathrm{E}$

\title{
Parton-Parton Elastic Scattering and Rapidity Gaps at Very High Energies ${ }^{\star}$
}

\author{
Vittorio Del Duca and Wai-Keung TAng \\ Stanford Linear Accelerator Center \\ Stanford University, Stanford, California 94309
}

\begin{abstract}
The theory of the perturbative pomeron, due to Lipatov and collaborators, is used to compute the probability of observing parton-parton elastic scattering and rapidity gaps between jets in hadron collisions at very high energies.
\end{abstract}

Submitted to Physics Letters B

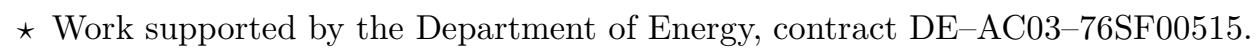


The energies of the Tevatron, LHC and SSC colliders open a window on a range of events in a new kinematical regime of hadron scattering, the semihard regime, where the hadron center-of-mass energy $\sqrt{s}$ is much larger than the typical parton transverse momentum $m$. This complicates the experimental analysis of such events, since it requires jet detection in a large rapidity interval ${ }^{[1]}$. It also makes harder the calculation of jet cross sections, since it involves large ratios of kinematical invariants which may appear either in the short distance cross section ${ }^{[2]}$ or in the evolution of the parton structure functions ${ }^{[3]}$, thus requiring the introduction of techniques to resum infinite classes of Feynman diagrams. Nonetheless the study of jet cross sections at very high energies is very interesting, since it may provide new insights on the dynamics of QCD processes and offer signatures of novel physical events ${ }^{[4]}$.

In order to obtain quantitative predictions of jet production in the semihard regime and separate it from the uncertainty involving the small $x$ dependence of parton distributions ${ }^{[3]}$, Mueller and Navelet ${ }^{[2]}$ proposed to measure the two-jet inclusive cross section in hadron collisions by tagging two jets at a large rapidity interval $y$ and with transverse momentum of order $m$. These tagging jets are produced in a nearly forward scattering of gluons or quarks with large center-ofmass energy $\sqrt{\hat{s}}$. Lipatov and collaborators ${ }^{[5-8]}$ (BFKL) have shown that, in this regime, the rapidity interval $y=\ln \left(\hat{s} / m^{2}\right)$ between the scattered partons is filled in by the radiation of additional gluons, roughly uniformly spaced in rapidity, all with transverse momenta of order $m$. The BFKL theory systematically corrects the lowest-order QCD result by summing the leading logarithms of $\hat{s}$. This is done in three stages, as shown in Fig. 1. First, one simplifies the lowest-order QCD diagrams for multigluon production, shown in Fig. 1(a), for the case in which the emitted gluons are widely separated in rapidity. The gluon emission vertex is replaced by a non-local gauge-invariant effective vertex ${ }^{[5]}$. Next, one sums the leading corrections to the forward amplitude with color octet exchange in the $t$ channel, as shown in Fig. 1(b). The result has the form of a Regge pole with an infrared-sensitive trajectory ${ }^{[6]}$. Finally, one uses this resummed, effective gluon 
exchange to compute the elastic amplitude in the Regge limit $\hat{s} \gg-t$ with color singlet exchange in the $t$-channel, as shown in fig.1(c). This is known as the BFKL pomeron $^{[7]}$. The imaginary part of the forward amplitude is the parton-parton total cross section. To leading order in rapidity, the parton-parton total cross section and the related 2-jet inclusive cross section exhibit the energy dependence $\exp \left[\left(\alpha_{P}-1\right) y\right]$ with

$$
\alpha_{P}=1+4 \ln 2 \frac{\alpha_{s} C_{A}}{\pi}
$$

where $C_{A}=N_{c}=3$ is the number of colors in QCD.

A large total cross section $\hat{\sigma}_{\text {total }}$ for parton-parton scattering implies also a large elastic scattering cross section, since the total cross section is related, through the optical theorem, to the elastic scattering amplitude with color singlet exchange in the $t$ channel. Thus both elastic scattering and jet production can be described by exchanging one BFKL pomeron Fig. 2(a,b). This process has been studied by Mueller and one of the authors ${ }^{[9]}$. It is a higher order $\left(\alpha_{s}^{4}\right)$ process but with energy dependence $\exp \left[2\left(\alpha_{P}-1\right) y\right]$. However, it leads to a final state which, at the parton level, contains two jets with a rapidity gap in gluon production between them ${ }^{[4]}$. Some fraction of these states may produce the dramatic experimental signatures of a large rapidity gap in secondary particle production. To understand the relation between rapidity gaps in hard-gluon and hadron production, we must discuss the potential backgrounds to these signals at the parton and hadron level.

To analize the parton-level background, assume that we cannot detect partons with transverse momentum smaller than a fixed parameter $\mu$. In this case, there is an additional contribution to elastic scattering from color octet exchange in the $t$ channel. This is shown in fig.1(b) where a reggeized gluon is exchanged. The reggeized gluon contains all the leading virtual radiative corrections and it has the form of a Sudakov form factor. The parameter $\mu$ fixes the scale below which soft gluon radiation is allowed. As $\mu \rightarrow 0$, the contribution of the color octet exchange vanishes, since it is impossible to have scattering with exchange of a gluon, without allowing for the emission of soft gluon radiation. 
In order to use perturbative QCD, the parameter $\mu$ must be larger than $\Lambda_{Q C D}$. Thus we have two options: first, we can consider $m \gg \mu \gg \Lambda_{Q C D}$, that is, we define a rapidity gap to be present if there are no jets between the tagging jets. We will call this case quasi elastic scattering, since it allows gluon radiation below the scale $\mu$. The ratio $R$ of the quasi-elastic to the total cross section is given by

$$
R(\mu)=\frac{\sigma_{\text {singlet }}+\sigma_{\text {octet }}}{\sigma_{\text {tot }}}
$$

where all the cross sections in (2) have been convoluted with the appropriate parton distributions. $^{[10]}$ Alternatively, we can consider $\mu=O\left(\Lambda_{Q C D}\right)$. Then at the parton level the color octet exchange is strongly suppressed, and only the color singlet exchange contributes to the cross section for producing rapidity gaps.

At the hadron level, there is an additional consideration. Accompanying any hard QCD reaction in hadron-hadron collisions, there are spectator partons. These may produce hadrons across the rapidity interval, spoiling the rapidity gap. Thus in order to compute the cross section for producing a rapidity gap at the hadron level, we need a non-perturbative model which describes the hadron interaction and estimates the survival of the rapidity gap in the presence of soft spectator interactions $^{[4]}$. The rapidity-gap survival probability $\left\langle S^{2}>\right.$ is defined as the probability that in a scattering event no other interaction occurs beside the hard collision of interest. This probability is most readily estimated as an average over the hadron-hadron impact parameter $B^{[4]}$ :

$$
<S^{2}>=\frac{\int d^{2} B f(B) S^{2}(B)}{\int d^{2} B f(B)}
$$

where $S^{2}(B)$ is the probability that the colliding hadrons do not interact inelastically, and $f(B)$ is the cross section for the hard collision of interest. Different estimates for $<S^{2}>$ in hadron collisions, based on a variety of phenomenological models, are presented in ref.10, where $\left\langle S^{2}>\right.$ is estimated to be between 0.05 and 0.2. $<S^{2}>$ is expected to depend on the hadron-hadron center of mass energy, 
but only weakly on the size of the rapidity gap. Then to obtain the probability of a scattering event with a large rapidity gap at the hadron level, we must compute the ratio $R$ at $\mu=0$, that is, using only the singlet elastic cross section, and multiply it by the survival probability $<S^{2}>$ :

$$
R_{\text {gap }}=<S^{2}>R(\mu=0)
$$

In this paper, we will present the asymptotic form of $R(\mu)$, and a numerical estimate for this quantity for rapidity intervals $y<15$.

For very large rapidity intervals, the one pomeron exchange approximation violates unitarity since the elastic cross section becomes larger than the total cross section. Our estimate indicates that the unitarity bound for the one pomeron exchange approximation is at about 24 units of rapidity, when the transverse momentum of the tagging jets $m$ is about $20 \mathrm{GeV}$. This is far beyond the maximum rapidity interval available at the SSC. At lower jet transverse momenta or for larger rapidity intervals, $R$ must be unitarized by the contribution from multiple pomeron exchange.

\section{Total Cross Section for Tagging Jets}

The total cross section for two tagging jets has been studied by Mueller and Navelet $^{[2]}$, who used the BFKL analysis of the asymptotic parton-parton total cross section to derive the 2-jet inclusive cross section. Following the exposition of ref. 11 , we consider the scattering of two hadrons of momenta $p_{A}$ and $p_{B}$ in the centerof-mass frame, with the $z$ axis along the beam momenta, and we imagine to tag two jets at the extremes of the Lego plot, with the rapidity interval between them filled with jets. We will call these extra jets minijets. The tagging jets can be characterized by their transverse momenta and by their longitudinal fractions $x_{A}$, $x_{B}$ with respect to their parent hadrons. It is simplest to consider the cross section for producing two tagging jets with transverse momenta greater than a minimum 
value $m$. Then

$$
\begin{aligned}
\frac{d \sigma_{t o t}}{d x_{A} d x_{B}}\left(A B \rightarrow j\left(x_{A}\right) j\left(x_{B}\right)+X\right) & \\
& =\prod_{i=A, B}\left[G\left(x_{i}, m^{2}\right)+4 / 9 \sum_{f}\left[Q_{f}\left(x_{i}, m^{2}\right)+\bar{Q}_{f}\left(x_{i}, m^{2}\right)\right]\right] \cdot \hat{\sigma}_{t o t}(\hat{s}),
\end{aligned}
$$

where $\hat{s}=2 p_{A} \cdot p_{B} x_{A} x_{B}$ is the parton-parton squared center-of-mass energy, and $\hat{\sigma}_{\text {tot }}$ is the BFKL total cross section for gluon-gluon scattering within an impact distance of size $1 / m$. Eq. (5) includes the effects of quarks using the observation of Combridge and Maxwell that, in a process with large rapidity intervals, the leading contribution to any scattering process comes from gluon exchange in the crossed channel $^{[12]}$. The factor $4 / 9$ in eq.(5) is the ratio of the Casimir operators $C_{F} / C_{A}$, with $C_{F}=\left(N_{c}^{2}-1\right) / 2 N_{c}$. The values of $x_{A}, x_{B}$ should be taken to be sufficiently large that the parton distributions satisfy ordinary DGLAP (Dokshiter, Gribov, Lipatov, Altarelli and Parisi) evolution; the BFKL theory adds additional complications in the evolution of the parton structure functions when these fractions become small ${ }^{[3]}$.

The core of eq. (5) is the BFKL function $\hat{\sigma}_{t o t}(\hat{s})$, which is given by

$$
\hat{\sigma}_{t o t}(\hat{s})=\frac{8}{N_{c}^{2}-1}\left(\frac{\alpha_{s} C_{A}}{\pi}\right)^{2} \frac{\pi^{3}}{2 m^{2}} F_{t o t}(y) .
$$

$F_{\text {tot }}(y)$ is a dimensionless function which we will discuss below. The strong coupling constant is evaluated at a scale $m^{2}$; the running of $\alpha_{s}$ is subleading in the BFKL theory. $F_{t o t}$ is normalized to 1 at the lowest order of QCD for the gluon-gluon scattering cross section, integrated over the transverse momenta of the tagging jets with $\left|k_{i \perp}\right| \geq m$, in the limit $\hat{s}>>m^{2}$. Since $F_{t o t}$ is always larger than 1 , it can be viewed through the optical theorem as the enhancement factor due to pomeron exchange. $F_{\text {tot }}$ can be calculated from the solution of an integral equation for the elastic scattering amplitude, with color singlet exchange, constructed by BFKL ${ }^{[6-8]}$. It is given by ${ }^{[2]}$ 


$$
F_{t o t}(y)=\int_{-\infty}^{\infty} \frac{d \nu}{2 \pi} \frac{1}{\nu^{2}+1 / 4} e^{\omega(\nu) y}
$$

with

$$
\omega(\nu)=\omega(0, \nu)
$$

the value at $n=0$ of the eigenvalue of the integral equation

$$
\omega(n, \nu)=\frac{2 \alpha_{s} C_{A}}{\pi}\left[\psi(1)-\operatorname{Re} \psi\left(\frac{|n|+1}{2}+i \nu\right)\right]
$$

and $\psi(z)$ the standard logarithmic derivative of the Gamma function. The exponential growth of $F_{t o t}(y)$ with the rapidity interval is associated with minijet production. Near $\nu=0, \omega(\nu)$ has the expansion

$$
\omega(\nu)=A-B \nu^{2}+\cdots
$$

with

$$
A=\frac{4 \alpha_{s} C_{A}}{\pi} \log 2, \quad B=\frac{14 \alpha_{s} C_{A}}{\pi} \zeta(3)
$$

Using (10) to expand about the saddle point at $\nu=0$, we can see that $F_{\text {tot }}(y)$ has the asymptotic behavior

$$
F_{t o t}(y) \sim \frac{e^{(4 \log 2) z}}{\sqrt{\frac{7}{2} \zeta(3) \pi z}}, \quad \text { with } z=\frac{\alpha_{s} C_{A}}{\pi} y
$$

Mueller and Navelet showed that this asymptotic form is an accurate representation of eq.(7) for $z>0.2$.

\section{Elastic Cross Section for Tagging Jets}


The high energy elastic scattering cross section with color singlet exchange in the $t$ channel has been studied in ref. 9. Then it is straightforward to write down the elastic cross section for tagging jets, with singlet exchange, as

$$
\begin{aligned}
\frac{d \sigma_{\text {singlet }}}{d x_{A} d x_{B}}\left(A B \rightarrow j\left(x_{A}\right) j\left(x_{B}\right)\right) & \\
& =\prod_{i=A, B}\left[G\left(x_{i}, m^{2}\right)+(4 / 9)^{2} \sum_{f}\left[Q_{f}\left(x_{i}, m^{2}\right)+\bar{Q}_{f}\left(x_{i}, m^{2}\right)\right]\right] \cdot \hat{\sigma}_{\text {singlet }}(\hat{s}) .
\end{aligned}
$$

The BFKL function $\hat{\sigma}_{\text {singlet }}(\hat{s})$ is the gluon-gluon elastic scattering cross section in the color singlet channel with the tagging jets collimated and with minimum jet transverse momentum $m$. Since two reggeized gluons are involved in the color singlet exchange in the $t$ channel, in keeping into account the possibility that the scattering is initiated by quarks we obtain the suppression factor $\left(C_{F} / C_{A}\right)^{2}$. $\hat{\sigma}_{\text {singlet }}(\hat{s})$ is given by

$$
\hat{\sigma}_{\text {singlet }}(\hat{s})=\left(\frac{8}{N_{c}^{2}-1}\right)^{2}\left(\frac{\alpha_{s} C_{A}}{\pi}\right)^{4} \frac{\pi^{5}}{4 m^{2}} F_{\text {singlet }}(y)
$$

with

$$
F_{\text {singlet }}=\frac{m^{2}}{16 \pi^{3}} \int_{m^{2}} d^{2} k_{A \perp}\left|\int d^{2} q_{\perp} d^{2} q_{\perp}^{\prime} f^{k_{A \perp}}\left(q_{\perp}, q_{\perp}^{\prime}, y\right)\right|^{2}
$$

The amplitude $f^{k_{A \perp}}\left(q_{\perp}, q_{\perp}^{\prime}, y\right)$ represents the propagation of a BFKL pomeron in transverse momentum plane (fig.2(b)), with $t=-k_{A, \perp}^{2}$. The normalization of $f^{k_{A \perp}}\left(q_{\perp}, q_{\perp}^{\prime}, y\right)$ is as in ref. 8 so that at lowest order

$$
f^{k_{A \perp}}\left(q_{\perp}, q_{\perp}^{\prime}, y\right)=\frac{\delta^{2}\left(q_{\perp}-q_{\perp}^{\prime}\right)}{q_{\perp}^{2}\left(q_{\perp}^{\prime}-k_{A \perp}\right)^{2}}
$$

Lipatov $^{[7,8]}$ gives an integral representation of $f^{k_{A \perp}}\left(q_{\perp}, q_{\perp}^{\prime}, y\right)$ in impact param- 
eter space, for the scattering of color neutral objects. The extension to partonparton scattering has been done in ref.9. The integral of $f^{k_{A \perp}}\left(q_{\perp}, q_{\perp}^{\prime}, y\right)$, over its transverse momenta, is ${ }^{[9]}$

$$
\int d^{2} q_{\perp} d^{2} q_{\perp}^{\prime} f^{k_{A \perp}}\left(q_{\perp}, q_{\perp}^{\prime}, y\right)=\frac{4}{k_{A \perp}^{2}} \int d \nu \frac{\nu^{2}}{\left(\nu^{2}+\frac{1}{4}\right)^{2}} \exp [\omega(\nu) y],
$$

where only the leading term $n=0$, for which $\omega(\nu)$ is given by (8) and (9), has been kept. As Mueller and Tang have shown, the integration over the transverse momenta in (17) is not infrared sensitive, i.e. the infrared divergences in $q_{\perp}$, which are present in the lowest order contribution (16), disappear when we consider the infinite resummation (17) of the leading logarithmic corrections. It is an interesting result that the pomeron trajectory has no explicit dependence on the momentum transfer. The dependence on $t$ is only through the coupling constant.

By substituting (17) into (15) we calculate $F_{\text {singlet }}$. The integral over $k_{A \perp}$ is singular and depends on the cutoff $m$; this gives us the same factor $m^{-2}$ as in the total cross section. The enhancement factor becomes

$$
F_{\text {singlet }}(y)=4\left(\int \frac{d \nu}{2 \pi} \frac{\nu^{2}}{\left(\nu^{2}+\frac{1}{4}\right)^{2}} \exp [\omega(\nu) y]\right)^{2}
$$

$F_{\text {singlet }}$ is normalized in order to have $F_{\text {singlet }}=1$ at $y=0$. Notice, though, that $F_{\text {singlet }}$ at $y=0$ from (18) does not correspond to gluon-gluon elastic scattering at the lowest order of QCD, which is instead correctly given substituting (16) into (15). Using the expansion of $\omega(\nu)$ around the saddle point $\nu=0$, we obtain the asymptotics of $F_{\text {singlet }}$

$$
F_{\text {singlet }}(y) \sim\left(\pi \frac{e^{(4 \log 2) z}}{\left(\frac{7}{2} \zeta(3) \pi z\right)^{3 / 2}}\right)^{2}
$$

where $z$ is defined in (12). 
The background to the color singlet exchange comes from the exchange of a reggeized gluon. This contribution is

$$
\begin{aligned}
\frac{d \sigma_{\text {octet }}}{d x_{A} d x_{B}} & \left(A B \rightarrow j\left(x_{A}\right) j\left(x_{B}\right)\right) \\
& =\prod_{i=A, B}\left[G\left(x_{i}, m^{2}\right)+4 / 9 \sum_{f}\left[Q_{f}\left(x_{i}, m^{2}\right)+\bar{Q}_{f}\left(x_{i}, m^{2}\right)\right]\right] \cdot \hat{\sigma}_{o c t e t}(\hat{s}, \mu),
\end{aligned}
$$

$\hat{\sigma}_{\text {octet }}(\hat{s}, \mu)$ is the gluon-gluon elastic scattering cross section in the color octet channel, with the tagging jets collimated and with minimum transverse momentum $m$. We may write $\hat{\sigma}_{\text {octet }}(\hat{s}, \mu)$ as ${ }^{[9]}$

$$
\hat{\sigma}_{\text {octet }}(\hat{s}, \mu)=\frac{8}{N_{c}^{2}-1}\left(\frac{\alpha_{s} C_{A}}{\pi}\right)^{2} \frac{\pi^{3}}{2 m^{2}} F_{\text {octet }}(y),
$$

where $F_{\text {octet }}$ is the solution of the BFKL integral equation for elastic scattering with color octet exchange ${ }^{[6]}$

$$
F_{\text {octet }}=\exp \left[2\left(\alpha_{g}-1\right) y\right]
$$

The reggeized gluon trajectory is

$$
\alpha_{g}=1-\frac{\alpha C_{A}}{\pi} \log \left(\frac{m}{\mu}\right),
$$

for $m^{2} / \mu^{2} \gg 1$. From the expression of the gluon trajectory, we can see that $\alpha_{g}-1<0$ and hence $F_{\text {octet }}$ is always less than one. As $\mu \rightarrow 0$, or the rapidity interval $y$ becomes large, $\hat{\sigma}_{\text {octet }}(\hat{s}, \mu)$ vanishes.

The Ratio $R(\mu)$

$R(\mu)$ is the probability of having elastic scattering at the parton level, as defined 
in (2). By substituting (20), (13) and (5) into the definition of $R(\mu)$, we obtain

$$
R\left(x_{A}, x_{B}, \mu\right)=\frac{\hat{\sigma}_{\text {octet }}}{\hat{\sigma}_{\text {tot }}}+w_{f}\left(x_{A}\right) w_{f}\left(x_{B}\right) \frac{\hat{\sigma}_{\text {singlet }}}{\hat{\sigma}_{\text {tot }}}
$$

where $w_{f}$ is a weight of parton distributions,

$$
w_{f}(x)=\frac{\left[G\left(x, m^{2}\right)+(4 / 9)^{2} \sum_{f}\left[Q_{f}\left(x, m^{2}\right)+\bar{Q}_{f}\left(x, m^{2}\right)\right]\right]}{\left[G\left(x, m^{2}\right)+(4 / 9) \sum_{f}\left[Q_{f}\left(x, m^{2}\right)+\bar{Q}_{f}\left(x, m^{2}\right)\right]\right]}
$$

and

$$
\begin{aligned}
\frac{\hat{\sigma}_{\text {singlet }}}{\hat{\sigma}_{\text {tot }}} & =\frac{8}{N_{c}^{2}-1}\left(\frac{\alpha_{s} C_{A}}{\pi}\right)^{2} \frac{\pi^{2}}{2} \frac{F_{\text {singlet }}}{F_{\text {tot }}} \\
\frac{\hat{\sigma}_{\text {octet }}}{\hat{\sigma}_{\text {tot }}} & =\frac{F_{\text {octet }}}{F_{\text {tot }}} .
\end{aligned}
$$

In the asymptotic regime, where the rapidity $y$ is large,

$$
\begin{aligned}
\frac{F_{\text {singlet }}}{F_{\text {tot }}} & \sim \pi^{2} \frac{e^{(4 \log 2) z}}{\left(\frac{7}{2} \zeta(3) \pi z\right)^{5 / 2}} \\
\frac{F_{\text {octet }}}{F_{\text {tot }}} & \sim e^{-(4 \log 2+2 \log (m / \mu)) z} \sqrt{\frac{7}{2} \zeta(3) \pi z}
\end{aligned}
$$

and so the octet contribution can be neglected in the asymptotic regime. The asymptotic expressions can be used to estimate the range of validity of the one pomeron exchange approximation. Taking $\alpha_{s} \sim 0.15$, which is the typical value for tagging jets of transverse momentum $m=20 \mathrm{GeV}$, the violation of the unitarity bound happens at a rapidity interval $y \simeq 24$.

\section{Numerical Evaluation}

We now turn to a numerical analysis of $R(\mu)$. The formulae (7), (18), (22) and (24) are the appropriate starting point for a numerical evaluation of the ratio $R(\mu)$. We scale the running coupling constant from $\alpha_{s}(m(Z))=0.12$ using the 1-loop evolution with 5 flavors, and take the longitudinal fractions $x_{A}, x_{B}$ of the tagging jets to be 0.1 . 
In Fig. 3, we show the result for $m=10-40 \mathrm{GeV}$. Even though we present the result from $y=0$, the plots cannot be trusted in the small rapidity regime since the leading logarithmic approximation is no longer valid there. The dotted line is the contribution from color singlet exchange only, and thus corresponds to the choice $\mu=0$, while the solid line includes both singlet and octet exchanges. In Fig. 3(a), $\mu$ is set to $1 \mathrm{GeV}$. For $y>7$, only the color singlet contributes since the octet is highly suppressed. In Fig. 3(b), $\mu$ is set to $5 \mathrm{GeV}$. The suppression of the octet occurs now for $y>10$. The two figures show the same trend in the asymptotic regime $(y>10)$ since the singlet exchange is independent of the choice of $\mu$.

The value of $R(\mu=0)$, given by the dotted line in Fig. 3, multiplied by the survival propability $<S^{2}>(3)$, gives the probability of having a collision with a large rapidity gap (4). Bjorken ${ }^{[4]}$ has estimated $\left\langle S^{2}>\right.$ to be $\simeq 0.1$, and the authors of ref.10 have given estimates in the range of 0.05 to 0.2 . Thus, we expect that, at the Tevatron, a few tenths percent of events with tagging jets will show rapidity gaps in hadron production. The probability of finding a gap increases with the rapidity interval between the tagging jets, as indicated in the figure. Though $<S^{2}>$ depends on the collider center of mass energy, it should depend only weakly on rapidity. Thus, we expect that the probability of observing a rapidity gap in secondary particle production between tagging jets should rise proportionately to the dashed curves in Fig. 3.

Since all of the analysis above is in the leading logarithmic approximation, there is ambiguity in the choice of the proper scale in rapidity for which this analysis is valid, and so the exact value of the normalization and thus of $R(\mu)$ cannot be determined precisely. We need a next-to-leading order calculation, which is not available yet, to have a definite quantitative prediction. However, the slope of the curves in the asymptotic regime is free from this scale uncertainty and thus the experimental measurement of the ratio $R(\mu) / R_{\text {gap }}$ in the large rapidity-gap regime should give us an unambiguous determination of the survival probability $<S^{2}>$. It will be interesting to see if $<S^{2}>$ depends on the width of the rapidity gap 
between the tagging jets, when the center-of-mass energy of the colliding hadrons is kept fixed.

\section{Acknowledgements}

We are very grateful to bj. Bjorken and Michael Peskin for encouraging us to investigate this problem, and for many stimulating discussions and suggestions, and for constructive criticisms while reading this manuscript. 


\section{REFERENCES}

1. J.D. Byorken, Int. J. Mod. Phys. A7 (1992) 4189.

2. A. H. Mueller and H. Navelet, Nucl. Phys. B282 (1987) 727.

3. J.C. Collins and R.K. Ellis, Nucl. Phys. B360 (1991) 3;

S. Catani, M. Ciafaloni and F. Hautmann, Nucl. Phys. B366 (1991) 135.

4. J. D. Bjorken, Phys. Rev. D47 (1992) 101.

5. L. N. Lipatov, Sov. J. Nucl. Phys. 23 (1976) 338.

6. E. A. Kuraev, L. N. Lipatov, and V. S. Fadin, Sov. Phys. JETP 44 (1976) 443, 45 (1977) 199.

7. Ya. Ya. Balitsky and L. N. Lipatov, Sov. J. Nucl. Phys. 28 (1978) 822;

L. N. Lipatov, Sov. Phys. JETP 63 (1986) 904.

8. L. N. Lipatov, in Perturbative QCD, ed. A.H. Mueller (World Scientific, Singapore, 1989).

9. A. H. Mueller and W.-K. Tang, Phys. Lett. B284 (1992) 123.

10. E. Gotsman, E.M. Levin and U. Maor, TAUP-2030-93 preprint.

11. V. Del Duca, M. E. Peskin and W.-K. Tang, SLAC-PUB-6065 preprint, to appear in Phys. Lett. B.

12. B. L. Combridge and C. J. Maxwell, Nucl. Phys. B239 (1984) 429. 


\section{FIGURE CAPTIONS}

1) BFKL resummation: (a) emission of gluons; (b) construction of the octet amplitude; (c) construction of the singlet amplitude.

2) BFKL Pomeron: (a) in minijet production; (b) in high energy elastic scattering.

3) Ratio $R(\mu)$ as a function of the rapidity $y$ of the tagging jets. We choose $x_{A}$ and $x_{B}$ to be 0.1 . The dotted line is the color singlet contribution. The solid line includes the contribution from both singlet and octet. We label the curves according to the minimum jet transverse momentum. (a) $\mu=1 \mathrm{GeV}$; (b) $\mu=5 \mathrm{GeV}$. 